parmi lesquels il faut eiter MM. Exmer, Claessen, Ashbrook, fascher, elc., nous possédons actuellement le moyon d'abréger dans une notable mesure le temps nécessarre à une analyse dectrolytique. Un dosage de cuivre peut être terminé en 10 ou 15 minutes, un peu plus survant les cas. Il suffit pour cela d'anmer d'un mouvement de rotation rapide l'électrode oppostéc is celle de dépot ; l'anode s'il s'agıt d'un dépot cathodıque, la calhode dans le cas contraire. De nombreux dispositifs ont ćté imagnés pour exécuter commodément cette rotation. Lá méthode dannlyse rapıde se prête à des séparations variées et prtésenle nème, dans ce cas, l'avanlage de permettre un controle de la marelie pendant toute l'općration, pusque sa durće est énormément raccourcie. (Voir O. Donx-IIÉnaul.T, Bull. Soc. Chumique de Bilginue, mars 1905 et Electroanalytische-Schnelmethoden, par lo $\mathrm{V}^{\mathrm{r}}$ A. Fischer, Stultgart, 1908).

\section{LE FOUR ÉLECTRIQUE DANS LA LOIRE}

Dans une très belle étude sur « L'Industrie métallurgique dans le bassin de la Loire ", que vient de publier le Bulletin de la Socrété de l'Industrie Minérale, M. A. Vicalre, ingénieur au Corps des Mines, s'exprime ainsi au sujet du four électrique :

«Notre région a suivi avec beaucoup d'intérêt le développement de l'emploi du four électrique, ce jeune rival du creuset qui se posera peut-être demain en concurrent du Martin, en auxiliaire indispensable du convertisseur. On sait qu'en Allemagne le fer électrique a fait son entrée sur le marché des produits presque courants, et qu'il a été commandé plusieurs milliers de tonnes de rails en acier électrique. En France, ce nouveau venu n'a pas encore fait parade d'ambitions aussi vastes et il n'est pas sorti du domaine des aciers de qualité. La question de savoir si l'acier électrique est égal en tout point aux aciers au creuset est encore controversée, et dans la Loire on est loin d'admettre qu'il lui soit supérieur à composition identique, comme cela a été avancé récemment. L'excellence de l'acier au creuset provient en grande partie de l'élimination parfaite des scories; il n'est pas prouvé que cette élimination soit aussi satisfaisante avec les fours électriques actuels dont les dispositifs de coulée rappellent ceux du four Martin. Mais l'avenir de cet appareil si souple est illimité, et il a dès maintenant le grand avantage de se contenter de riblons ordinaires an lieu d'exiger comme le creuset des matières exceptionnelles, dont le prix s'élève parfois, quand il s'agit de certains fers de Suède, à 50 ou $60 \mathrm{fr}$. les roo kilogs

"La maison Holtzer, dont le nom occupe une si grande place dans les annales du creuset, a mis en marche, il y a quelques semaines, un four Keller de io to nes, le plus grand four électrique qui soit actuellement en marche dans le monde entier; il est caractérisé par l'emploi de deux paires d'électrodes; un moteur de 750 kilowatts lui fournit son courant. Suivant la formule actuellement en faveur chez nous, et qui s'impose quand le courant électrique est cher, ce four sert à épurer de l'acier déjà phosphoré et décarburé dans un four Martin de même capacité. Les résultats obtenus ont été satisfaisants, mais on n'a encore publié aucun renseignement économique.

(c. MM. Marrel ont étudié le même problème avec la Société Girod, dont on connait le four d'une simplicité si remarquable. Les aciéries de Firminy l'ont abordé d'un manière indépendante, et ont construit un four d'essai de roo kg. Ce four n'a quiune électrode, sa sole en magnésie contient assez de charbon pour être un peu conductrice à froid sans que l'acier risque d'être carburé, le courant l'échauffe en passant et la rend conductrice, comme le filament d'une lampe Nernst. Les résultats des essais sont très encourageants (i).

"Quant aux Aciéries de la Marine, leurs essais remontent déjà à une épuque assez ancienne. Cette Société a toujours su

(1) M. Dumuis, directeur-adjoint des Aciéries de Firminy, a exposé ces resultats dans la Revue de Métallurgie (octobre 1908). localiser ses fabrications là où les conditions qu'elles exigcaient se trouvaient réalisées de la manière la plus avantageuse. La création du Boucau, l'achat d'Homécourt, en sont des preuves éclatantes. Elle envisagerait plutôt l'établissement d'une aciérie électrique dans le Nauphiné : on peut, en effet, s'y procurer le kilowalt-heure pour $1 / 2$ à I centime, tandis que, dans la Loire, les machines à vapeur ne peuvent guère le livrer pour moins de 5 à 6 centimes ( 1 ). L'aciérie des Alpes tıaiterait directement des riblons froids, et expédierait ensuite ses lingots aux forges de la Loire. Comme les lingots d'acier fin sont généralement refroidis et burinés avant le forgeage, l'éloignement de l'acićrie et de la forge n'entraînerait pas une dépense supplémentaire de réchauffage. C'est dans ces conditions que travaille actuellement l'aciérie électrique de la Socićté Girod, à llgines, aciérie que des agrandissements en cours d'exécution vont placer au premier rang des entreprises similaires (2) 》.

\section{BÈRLEMEHTATION DES DISTRIBUTIONS D'ÉEERQIE}

\section{CIRCULAIRE DU MINISTRE DES TRAVAUX PUBLICS DU 13 MARS 1909 RELATIVE AUX FRAIS DE CINTROLE}

A Monsieur l'Ingénieur en chef du Contróle des distributions d'énergie électrique.

En signalant à l'Administration les difficultés auxquelles donne lieu, dans leur service, la préparation de l'ćtat de remboursement des frais de contrôle dus à l'Etat, en vertu du décret du 17 octobre 1907 , par les permissionnaires ou concessionnaires des distributions d'énergie électrique, plusieurs ingénieurs en chef ont demandé à être fixés sur les points suivants :

$1^{\circ}$ Nonobstant les résistances des entrepreneurs de distributions d'énergie, les distributions établies antérieurement au décret du 17 octobre 1907 sont-elles soumises au versement des frais de contrôle?

$2^{\circ}$ Quel est le point de départ desdits frais pour ces distuibutions?

Il me paraît utile de porter à votre connaissance la décision que j'ai prise à ce sujet, sur l'avis de la Commission des distributions d'énergie élecırique.

En ce qui concerne la première question posee, il y a licu de distinguer les distributions établies par permission de voirie ou en vertu de concessions, et les frais de contrôle dus à l'Etat de ceux qui revieanent aux communes.

Les frais de contrôle constituent une taxe nouvelle créćc par la loi du 15 juin i 906 et immédiatement applicable à l'industric électrique comme le contrôle lui-même qu'elle a pour objet de rémunérer. Ils sont, par suite, exigibles pour toutes les cutreprises concédées ou munies de permissions de voiric, qu'elles soient antérieures ou non à la loi du i 5 juin rgo6, el ce, dans les conditions suivantes:

a. Si les distributions sont établies par permissions de voirie, les frais sont dus à l'Etat et aux communes.

$b$. Si les distributions sont installées en vertu de concessions, la part des frais dus à l'Etat doit toujou is être perçue.

En ce qui concerne les communes, deux cas se présentent: Ou bien l'acte de concession n'a rien spécifié à l'égard des frais de contrôle. Dans ce cas, les communes sont en droit d'en poursuivre le recouvrement dans les conditions fixées à l'article I I du décret du i 7 octobre 1907 .

Ou bien le cahier des charges a déterminé les frais de contrôle. Dans ce cas le contrat intervenu doit recevoir son plein effet, et les frais qui y sont inscrits doivent être maintenus purement et simplement, quand bien même la perception stipuléc au profit des communes serait supérieure à la perieption autorisée par l'article I I du décret susvisé.

(1) On pourrait probablement abaisser ce prix de revient en amenant du courant des Alpes.

(2) Elle sera aussi pourvue de laminoirs. 
En ce qui regarde la seconde question, il y a lieu de considérer que, à la date du 28 novembre 1907 , un arrêté de principe a chargé les ingénieurs en chef du service ordinaire de chaque département d'exercer le contrôle des distributions d'énergic ćlectrique, et qu'ainsi les services de contrôle ont été organisés avant le $1^{\mathrm{er}}$ janvier $\mathrm{I} g \mathrm{0}$. On pell donc adopter uniformément cette date comme point de départ de la taxation des frais de contrôle.

Le taux à appliquer pour chaque ligne doit être tixé :

a. En ce qui concerne les distributions postérieures à la loi, par le régime d'autorisation de chaque ligne, en conformité des prescriptions de la circulaire du 15 septembre 1908 .

b. En ce qui concerne les distributions anterieures à la loi, par le régime d'autorisation que la ligne aurait si on lui faisait application des principes posés par la loi du 5 juin Igo6 et par le décret du 17 octobre 1907 sur les frais de contrôle.

Louis Barthou. .

\section{CIRCULAIRE DU MINISTRE DES TRAVAUX PUBLICS DU 16 MARS 1909 RE! ATIVE AUX REDEVANCES DUES POUR L'OCCUPATION DU D SMAINE PUBLIC.}

A Monsieur l'ingénieur en chef du Contrôle des distributions d'énergie électrique.

Consulté à diverses reprises au sujet de 'application du décret du 17 octobre 1907 , portant fixation des redevances di es pour l'occupation du domaine public, j'ai provoqué, de la part de la Commission des distributions d'énergie ćlectrique, un avis dans lequel la question des redevances serait envisagée dans son ensemble.

J'al l'honneur de vous adresser ci-dessous copie de cet avis que j'ai adopté, et auquel vous devrez vous référer à l'occasion.

La Commission, considérant que, pour déterminer les redevances dues pour l'occupation du domaine public par des distributions d'énergie électrique, il y a lieu de distinguer les entreprises établies avant ou après la promulgation de la loi du 15 juin 1898 :

En ce qui concerne les entreprises établies postérieurement à la loi du 5 juin 1.906 :

Considérant que les redevances dues par ces entreprises sont fixées par le décret du i 7 octobre 1907 (Redevances), et que les mêmes règles sont applicables à toutes les canalisations, qu'elles soient aériennes ou souterraines, avec ou sans points d'appui sur le domaine public;

En ce qui concerne les entreprises établies antérieurement à la loi du 15 juin 1 go 6 :

Considérant que les permissions et concessions accordées par des actes antérieures à la loi sont maintenues dans leur forme et teneur par l'aricle 26 de la loi du 15 juin 1906 , et que les redevances fixées par le décret du i 7 octobre igo7 (Redevances) ne seront applicables aux distributions établies en vertu de concessions qu'à l'expiration de ces concessions, et aux distributions établies par permissions de voirie dès l'époque où les conditions fiscales de ces permissions seront susceptibles d'être revisées (art. 6 du décret du i 7 octobre i 907 ) (Redevances);

Considérant, d'autre part, que les concessions accordées par les communes antérieurement à la loi du 15 juin 1906 ne créaient au profit des concessionnaires aucun droit en dehors du domaine public communal, c: notamment sur le domaine public national et départemental;

Que l'occupation du domaine public national ou départemental ne pouvait avoir lieu qu'en vertu de permissions de voirie dont les communes rétrocédaient le bénéfice à leurs concesionnaires (Circulaires ministérielles du i 5 août et I $^{\text {er }}$ septembre I 893 ); que, dès lors, ces permissions de voirie, bien que régulièrement données aux communes pour être utilisées par leurs concessionnaires, restent soumises aux règlas générales qui sont applicables aux permissions de voirie accordées antérieurement à la loi ;

Est d'avis qu'il y a lieu de répondre dans le sens des observations ci-après :

\section{A. Distributions établies avant la promulgation de la loi du 15 juin 1906}

$1^{\circ}$ Distributions établies en vertu de permissions de volme

I. Sur la grande voirie nationale ou departementale. - L redevances fixées par le décret du 17 octobre 1907 sont applicables dès l'époque où les conditions fiscales de ces permissions sont susceptibles d'ètre revisées, c'est-à-dire :

a. Aux dates fixées par les arrêtés d'autorisation sur la grande voirie nationale ou départementale, lorsque ses arrêtés ont prévu cette revision;

b. Ou, lorsqu'ils n'ont fixé aucune date, à l'expiration du délai de 5 ans résuliant de l'application de l'article 4 de l'arrêtét interministériel du 3 août 1878 , pour la grande voirie natio. nale, ou du délai qui peut être fixé par l'article 26 de l'artêtét réglementaire du i 5 septembre i 893 , pour les routes départe. mentales.

II. Sur des voies faisant partie du domaine public communal. $-1^{\circ} \mathrm{Si}$ les permissions de voirie comportent des redevances, il y a lieu de les appliquer jusqu'à l'époque où les conditions fiscales de ces permissions sont susceptibles d'être revisées, c'est-à-dire aux dates fixées par les arrêtés d'autorisation, lorsque ces arrêtés ont prévu cette revision;

$2^{\circ} \mathrm{Si}$ les permissions de voirie ne comportent ni conditions, ni délais pour la revision des redevances, la loi du 15 juin rgó et ses annexes, notamment le décret du 17 octobre 1907 (Redfvances), n'apportent aucune moditicalion aux permissions de voirie accordées antérieurement.

\section{$2^{\circ}$ Distributions Établits en vertu de concessions}

III. Concessions communales accordées avant la promulgation de la loi du 15 juin igo6. - a. Lorsqu'elles ne comportent pas de canalisations autorisées par permission de voirie sur les dépendances de la grande voirie nationale ou départementale, aucune redevance n'est due à l'Etat: le régime fixé par le cahier des charges de la concession pour les redevances dues á l'autorité concédante est maintenu jus'qu'à l'expiration de ladite concession.

$b$. Lorsqu'elles comportent des canalisations autorisćes par permissions de voirie sur les dépendances de la grande voirie nationale ou départementale, ainsi que des canalisations établies, avec ou sans permissions de voirie, sur des voies faisant partie du domane public communal :

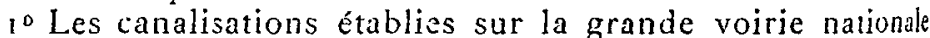
ou départementale sont soumises aux conditions de revisions ci-dessus fixées au paragraphe A, I ;

$2^{\circ}$ Les canalisations établies, avec ou sans permissions de voirie, sur les voies faisant partie du domaine public com. munal, restent soumises jusqu'à l'expiration de la concession au régime fixé pour les redevances dues à l'autorité concédante par le cahier des charges de ladite concession.

\section{B. Distributions établies après la promulgation de la loi du 15 juin 1906.}

IV. En ce qui concerne les distributions établies par permission de voirie ou en vertu de roncession des communes ou de l'Etat, postérieures à la promulgation de la loi du $x$ juin igob.

11 y a lieu de leur appliquer sans délai les redevances fixées par le décret du 17 octubre 1907.

Louis Barthov.

\section{FRAIS DE CONTROLE DES DISTRIBUTIONS D'ÉNERGIE}

Le Ministre des Travaux publics, Postes et Télégraphes,

Vu la loi du 5 juin 1906 sır les distributions d'énergie et notamment l'article i $8\left(3^{\circ}\right)$ portant qu'un règlement d'administration publique déterminera l'organisation du contrôle de la construction et de l'exploitation des distributions d'énergie électrique dont les frais sont à la charge du concessionnaire ot du permissionnaire;

Vu l'article 9 du décret du 17 octobre s 907 organisant ledit contrôle; 
Sur la proposition du Directeur du Personnel et de la Comp. abilité,

Arrête :

Les frais de contrôle dus à l'Etat par les Entrepreneurs de distributions d'énergie électrique, établies en vertu de permissions ou de concessions sont fixés, pour l'année r gog, à 1 o francs par kilomètre de ligne pour les distributions soumises au contröle des municipalités sous l'autorité du Ministre des Travaux publics des Postes et des Télégraphes.

Paris, le 23 avril 1909 .

Louis Barthov.

\section{LE MOIS HYDRO-ÉLECTRIQUE}

\section{ACADÉMIE DES SCIENCES}

\section{MÉCANIQUE ET ÉLECTRIGITÉ}

Des propriétés électriques des cupro-aluminiums (thermoélectricité et résistivité). Note de M. H. PÉchEux: Séance du 22 avril 1909 .

'Dans une précédente Note (8 mars Igog) j'ai exposé les résultats que f'a obtenus avec l'aluminum marchand, et montré l'influence des matières étrangères sur la thermo-électricité et la résistivité de ce métal. J'ai étudre ensuite six curro-aluminiums marchands, de fabricaion courante; lis m'ont fourni les résultals suivants, que jai lhontueur de communiquer à l'Acadómie.

${ }^{0}$ Thermo-électricate. - J'a fait un couple de chacun des alliages pobtenus en fils minces) avec le cutvre électrolytique. Chaque couple avat sa soudure disposée à côté de celle du couple pyrométrique nuckel-curvre bien étalonné; les soudures chaudes étaient portées lentement de la température ambiante à la température de $20^{0}$, le chauffage étant réalisé au four Mermet.

Les lectures obtenues pendant le refroidissement lent des soudures étaient seules conservées. La méthode suvie était toujours celle des déviations au galvanomètres Deprez-d'Arsonval avec règle de Poggendorff.

La force électromotrice de chaque couple suit une loi très sensiblement parabolıque, les écarts observés étant bien inférieurs aux erreurs possibles de leciure au galvanometre. L.es pouvoirs thermoelectriques de ces couples, calculés a l'aide du tableau des forces flectromotrices. peuvent être représentés par les formules suivantes (en microvolts).

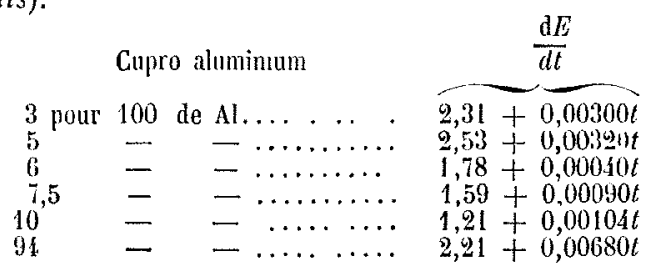

A l'aide de ces formules, on arrive à vérifier, conformément aux résultats fournis par l'expérimentation drecte, que :

a) Les alliages les plus riches en aluminium sont les plus éloignés, en général, de l'aluminium (dans la sérı thermo-électrique), exception faite pour l'alliage à 94 pour Ioo d'aluminium qui se rapproche davantage de ce métal:

b) Les alliages cupro-aluminiums ne suivent pas tous dans l'ordre deleur composition (contrairement aux conclusions de M. F. Steinmann, lequel d'ailleurs n'a étudié que les alliages à 5 pour roo, 7,5 pour 100 et io pour 100 , à trois températures seulement); ainsi en allant de l'aluminium au cuivre, dans la série, on trouve l'ordre sulvant (a 500 par exemple): 5 pour 100, 3 pour 100, 94 pour 100 , 6 pour $100,7,5$ pour 1 oo (ces deux derniers très voisins), et 10 pour 100. c) De $50^{\circ}$ a 8000 , il se produit quelques inversions modifiant l'ordre de la série; il y a inversion des alliages 3 pour roo et 94 pour 100 à 530 , des alliages 5 pour 100 et 94 pour 100 à 1780 , des alliages 6 pour 100 et 7,5 pour roo à 760 .

${ }^{20}$ Résistivlté. - J'ai mesuré la résistivité des six alliages, enroulés en spirale, bien recuits au préalable, en les portant à des températuses variables, dans un bain de paraffine, de la température ambiante à $350^{\circ}$; la méthode employee était celle du pont de Wheatsa corcle.

Les résultats obtenus sont consignés dans les formules suivantes, qui donnent la résistivité en micromhs-cm.

\begin{tabular}{|c|c|c|c|}
\hline \multicolumn{4}{|c|}{ Cupro-alummitum } \\
\hline pour & 100 & e $\Lambda 1$. & $q t=8,20\left(1+0.00102 t+0.000003 l^{2}\right)$ \\
\hline & - & & $p t=1021\left(1+0,00070 t+0,0000002 l^{2}\right)$ \\
\hline & - & & $P t=11,62\left(1+0,000055 t+0,000002 l^{2}\right)$ \\
\hline 3 & - & & $P l=18,62\left(1+0,00036 t+0,000001 t^{2}\right)$ \\
\hline & - & . & $f t=12,61\left(1+0,00032 t+0,000002 l^{2}\right)$ \\
\hline & - & & $=3,10$ \\
\hline
\end{tabular}

L'examen de ce tableau conduit aux inteıprétations suivantes:

a) La résistivité des cupro-aluminiums croît avec la teneur en aluminium jusqu'à 7,5 pour roo, alliage où elle est maxima; elle décroit ensuite; pour l'alliage à 94 pour 100 , elle se rapproche beaucoup de celle de l'aluminium métallique.

b) Le coefricient de température principal diminue quand la tencur en aluminium augmente, jusqu'a 10 pour roo. A 94 pour soo d'aluminium, il est voisı de celui de l'aluminium métallique.

c) L'didition de fables quantités d'alumınium au cuvre augmente très sensiblement la résistivité du cuivre; 1 l n'en est pas de même de l'addițion de faibles quantités de cuivre à l'aluminium; la résistivité de ce dernier nútal est à peine modifiée.

\section{SOCIÉTÉ INTERNATIONALE DES ÉLECTRICIENS}

\section{Séance du 5 mai 1909}

M. Pellat, présulent de la Société pour rgog, prononce le discours d'usage sur la Theorie electronique des courants et de leurs provriétes.

Puis M DE TRAz présente une communicition sur certaines condz. tions du probleme de la traction. Il montre que la "malche la plus économique doit comporter une accélération la plus forte possible départ, maintenue jusqu'à ce que la vitesse maxtma solt ittente; puis 11 faut une marche à vitesse constante, plus ou moins longue, suivant les conditions locales, ensuite une course sur l'erre, puis un freinage à accélération négative maxima jusqu'à l'arrêt ".

M. de Traz rappelle que la valeur de l'accélération, pendant le démarrage ou le freinage, est indifferente pour le confort des voya. geurs, zar, par suite du taible coefticient d'adherence des roues sur les rails, cette accélération ne peut pas pratiquement dépasser 2 métres par seconde par seconde. Ce qui incommode les voyageurs, c'est la variation brusque de l'accéleration. "L'effort devratcroitre an départ jusqu'à son maxımum, auquel il se mantiendrait jusqu’à ce que la vitesse approche de la vitesse maxima ").

\section{REVUE DES PÉRIODIQUES ÉTRANGERS}

Amélioration du facteur de puissance dans les distributions à courant alternatif. Milnes Walker, Llectrician, 22 janvier 1 gog.

L'auteur étudie lamélioration du facteur de puissance:

I $^{\circ}$ Par l'emploi de convertisseurs synchrones. Ce procédé est bien connu et nous n'y insistons pas;

$2^{\circ}$ Par l'emploi de capacilés: Ce procédé n'est point entré dans la pratique, en raison du prix élevé des condensateurs nécessaires, et pour lesquels la grande difficulté est l'absorption de la chaleúr produite dans le diélectrique;

3o Par perfectionnemint du moteur d'induction lui-même. La raison pour laquelle le moteur d'induction absorbe tant de courant dewatté, est que son courant magnétisant cst fourni à haute fréquence. Quand le courant magnétisant est fourni au rotor à basse fréquence des courants dans ce dernier, la puissance apparente magnétisante tombe à 2 ou 3 pour 100 de ce qu'elle est dans les conditions normales de fréquence. Après avoir rappelé les résultats de Leblanc et Heyland, l'auteur décrit une excitatrice speciale, dite « avanceur de phase » des. tiné à remplir ce but.

L'auteur termine en indiquant le moyen d'engager les consommateurs à améliorer leur facteur de puissance serait par une taxation basée sur les kilovoltampères absorbés, et non uniquement sur les kilowatts.

Méthode de mesure des très faibles inductances. C.-J. Watson. Electrician, Londres, 5 mars I gog.

Lorsqu'on expérimente avec de très rapides oscillations, il devient nécessaire de mesurer de très taibles inductances. Un moyen commode d'y arriver consiste à se servir d'une sorte de pont de Wheatstone, dans lequel deux inductances sont équilibrées par 2 capacités, avec cmploi d'un tube à vide au heu d'un galvanomètre comme appareil de zéro.

Le pont est alimenté en deux sommets par les armatures extérieures de deux bouteilles de Leyde, dont les armalutes intérieures sont connectées à une bobine d'induction capable de donner une etincelle de 5 centimetres. Comme inductance variable, on se sert de deux fils nustendus parallèlement, et dont un cavalier transversal permet d'intercaler en circuit une plus ou moins grande parile. 